\title{
Kelvin's chirality of optical beams
}

\author{
Sergey Nechayev $\odot,{ }^{1,2,3,4}$ Jörg S. Eismann, ${ }^{1,2,4,5}$ Rasoul Alaee $\oplus_{, 2,4}^{3,4}$ Ebrahim Karimi, ${ }^{1,3,4}$ \\ Robert W. Boyd $\odot, 3,4,6$ and Peter Banzer ${ }^{1,2,4,5, *}$ \\ ${ }^{1}$ Max Planck Institute for the Science of Light, Staudtstrasse 2, D-91058 Erlangen, Germany \\ ${ }^{2}$ Institute of Optics, Information and Photonics, University Erlangen-Nuremberg, Staudtstrasse 7/B2, D-91058 Erlangen, Germany \\ ${ }^{3}$ Department of Physics, University of Ottawa, 25 Templeton St., Ottawa, Ontario K1N 6N5, Canada \\ ${ }^{4}$ Max Planck-University of Ottawa Centre for Extreme and Quantum Photonics, 25 Templeton St., Ottawa, Ontario K1N 6N5, Canada \\ ${ }^{5}$ Institute of Physics, University of Graz, NAWI Graz, Universitätsplatz, 5, Graz, 8010 Austria \\ ${ }^{6}$ Institute of Optics and Department of Physics and Astronomy, University of Rochester, Rochester NY 14627 USA
}

(Received 17 December 2020; accepted 10 February 2021; published 2 March 2021)

\begin{abstract}
Geometrical chirality is a property of objects that describes a three-dimensional mirror-symmetry violation and therefore it requires a nonvanishing spatial extent. In contrary, optical chirality describes only the local handedness of electromagnetic fields and neglects the spatial geometrical structure of optical beams. In this Letter we put forward the physical significance of geometrical chirality of spatial structure of optical beams, which we term Kelvin's chirality. Furthermore, we report on an experiment revealing the coupling of Kelvin's chirality to optical chirality upon transmission of a focused beam through a planar medium. Our work emphasizes the importance of Kelvin's chirality in all light-matter interaction experiments involving structured light beams with spatially inhomogeneous phase and polarization distributions.
\end{abstract}

DOI: 10.1103/PhysRevA.103.L031501

\section{INTRODUCTION}

Since its first definition by Lord Kelvin in 1893 [1], the term "chiral" has found its use across the fields of physics, mathematics, chemistry, and biology. Chirality describes mirror-symmetry violation-if an object cannot be superimposed with its own mirror image by means of rotations and translations, it is termed chiral [1-4]. Consequently, geometrical chirality is inherently a nonlocal three-dimensional (3D) structural property of objects that requires a nonvanishing spatial extent [5-11]. On the other hand, optical chirality is a bit less tangible. Using parity inversion transform $\hat{\mathrm{P}}$ (a point reflection) [1-4] to define optical chirality allows electromagnetic beams to be chiral at specific points in space. The common definition of optical chirality as $C \propto \operatorname{Im}\left(\mathbf{E} \cdot \mathbf{H}^{*}\right)$ [12-21], where $\mathbf{E}$ and $\mathbf{H}$ refer to the electric and magnetic field vectors, respectively, is in perfect agreement with this.

Optical chirality plays a crucial role in chiral light-matter interactions [12,16,22-24]. Tools such as circular dichroism spectroscopy are widely used for distinguishing molecular enantiomers, studying proteins' structure, and measuring the composition of materials $[25,26]$. In all these scenarios, chiroptical phenomena are used to determine whether the

\footnotetext{
*peter.banzer@uni-graz.at
}

Published by the American Physical Society under the terms of the Creative Commons Attribution 4.0 International license. Further distribution of this work must maintain attribution to the author(s) and the published article's title, journal citation, and DOI. Open access publication funded by the Max Planck Society. interacting matter features geometrical chirality originating from its spatial extent.

However, polarization and phase distributions of optical beams can exhibit fascinating topological features in 3D space as well. These include knotted and linked polarization and phase singularities [27-29] and even polarization Möbius strips [30]. Some of these peculiar field topologies are structurally asymmetric upon parity inversion [31], rendering the optical beams geometrically chiral. Nonetheless, these beams do not necessarily also exhibit optical chirality $C$. Since the definition of $C$ only refers to a local arrangement of $\mathbf{E}$ and $\mathbf{H}$, it fails to describe any form of chirality originating from the spatial extent of the beams. This geometrical chirality of the spatial polarization and phase structure of optical beams (hereinafter referred to as Kelvin's chirality or $K$ ) may be directly involved in chiral light-matter interaction. Indeed, the corkscrew wave fronts of linearly polarized LaguerreGaussian beams $(C=0)$ readily engage in chiral light-matter interactions [32-39]. Also the polarization structure of vector beams with $C=0$ can produce circularly polarized light (CPL) upon scattering by achiral particles [40,41]. Kelvin's chirality therefore represents an electromagnetic equivalent of geometrical chirality and refers to a certain chiral arrangement of electromagnetic fields in 3D space. It complies with the point-reflection geometrical nonlocal definition of chirality, but it cannot be described involving optical chirality $C$.

To explore the phenomenon of Kelvin's chirality, we construct an experiment, where a normally incident polarizationstructured cylindrically symmetric beam with $C=0$ and $K \neq 0$ is focused and transmitted through a planar stratified medium. Surprisingly, we observe CPL in transmission $(C \neq 0)$ with its handedness being dependent on the incident 


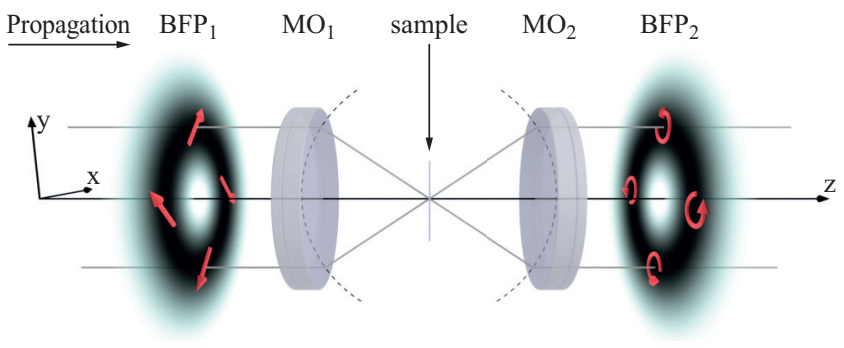

FIG. 1. Schematic illustration of the system. The incoming beam, propagating from left to right, is focused and collimated by two confocally aligned aplanatic microscope objectives (MOs). The sample is positioned normally to the $z$ axis between the MOs. The red arrows depict the local polarization of the beam before and after the introduction of a relative phase of $\pi / 2$ between radial and azimuthal polarization components.

$K$. We elucidate our results using a simple geometrical model, generalized Fresnel coefficients of the layered sample, and helicity conservation laws [17-21,42-49]. Our work calls for a careful evaluation of Kelvin's chirality as a crucial component of all light-matter interaction experiments that involve light beams with spatially inhomogeneous phase or polarization distributions.

\section{THEORY}

We investigate a system of two dry aplanatic microscope objectives $\left(\mathrm{MO}_{1}\right.$ and $\left.\mathrm{MO}_{2}\right)$ in confocal alignment, as sketched in Fig. 1, with an incident monochromatic beam propagating along the $z$ axis. Focusing by an aplanatic $\mathrm{MO}_{1}$ converts the spatial coordinates $\left(x_{1}, y_{1}\right)$ in the back focal plane $\left(\mathrm{BFP}_{1}\right)$ of $\mathrm{MO}_{1}$ to the angular coordinates of the focused beam via $k_{x}=$ $-x k / f, k_{y}=-y k / f$, where $f$ is the focal length, $k=2 \pi / \lambda$ is the wave number, and $\lambda$ is the wavelength [50]. Collimation by $\mathrm{MO}_{2}$ induces a reverse transformation such that in $\mathrm{BFP}_{2}$ we obtain $\left(x_{2}, y_{2}\right)=\left(k_{x} f / k, k_{y} f / k\right)$. To describe the evolution of the polarization pattern, we decompose the incident beam into radial and azimuthal polarization components, which correspond to the transverse magnetic (TM or $p$ ) and transverse electric (TE or $s$ ) polarization components of the focused beam, respectively. Reference [50] describes the process in detail.

Consider now a spirally polarized incident vector beam (see Fig. 1), which is an in- or $\pi$-out-of-phase superposition of a radially and an azimuthally polarized mode $[40,41,51,52]$. The electric field in the $\mathrm{BFP}_{1}$, which we assume to coincide with the beam's waist position $(z=0)$, can be written as

$$
\mathbf{E}_{\sigma}^{\text {in }}=E(\rho)[\hat{\boldsymbol{\rho}}+\sigma \hat{\boldsymbol{\varphi}}],
$$

where $E(\rho)=E_{0} \frac{\rho}{w_{0}} \exp \left(-\frac{\rho^{2}}{w_{0}^{2}}\right)$, with $w_{0}$ being the beam waist, $E_{0}$ is a constant, $\rho$ is the radial cylindrical coordinate, $\hat{\rho}$ and $\hat{\varphi}$ are the radial and axial unit vectors, respectively, and $\sigma= \pm 1$, corresponding to an in- or $\pi$-out-of-phase superposition. We note that parity transformation applied to the beam in Eq. (1) inverses the direction of the spiral polarization $\hat{\mathrm{P}}\left\{\mathbf{E}_{\sigma}^{\text {in }}\right\}=\mathbf{E}_{-\sigma}^{\text {in }}[31,41]$. Therefore, without assigning an exact value to $K$, we can argue that the beam in Eq. (1) possesses a nonzero Kelvin's chirality $K\left(\mathbf{E}_{ \pm \sigma}^{\text {in }}\right) \neq 0$ and that a pair beams with $\sigma= \pm 1$ constitute a pair of chiral enantiomers, which implies that $K\left(\mathbf{E}_{+\sigma}^{\text {in }}\right)=-K\left(\mathbf{E}_{-\sigma}^{\text {in }}\right)$. At the same time, such beams feature zero optical chirality $C$, which in the paraxial regime can be expressed as the third Stokes parameter $S_{3}$ [12-15,17-21]:

$$
C^{\text {in }}(\rho) \propto S_{3}^{\text {in }}(\rho)=2 \operatorname{Im}\left\{\left[\mathbf{E}_{\sigma}^{\text {in }} \cdot \hat{\boldsymbol{\rho}}\right]^{*} \cdot\left[\mathbf{E}_{\sigma}^{\text {in }} \cdot \hat{\boldsymbol{\varphi}}\right]\right\} \equiv 0 .
$$

We introduce a planar layered structure between the MOs (Fig. 1) positioned normally to the $z$ axis. Transmission through a stratified medium strongly depends on the polarization $(p, s)$ and angular coordinates $\left(k_{x}, k_{y}\right)$ of the focused beam. The angle of incidence of each angular component $\left(k_{x}, k_{y}\right)$ of the focused beam is defined by $\sin ^{-1}\left(k_{\rho} / k\right)$, where $k_{\rho}=\sqrt{k_{x}^{2}+k_{y}^{2}}$ [50]. Our sample is designed such that the Fresnel coefficients $t_{p}\left(k_{\rho}\right)$ and $t_{s}\left(k_{\rho}\right)$ for the transmitted $p$ - and $s$-polarized field components at an angle of $30^{\circ}$ with respect to the surface normal acquire a $\pi / 2$ phase difference, but have equal amplitudes $t_{p} / t_{s} \approx \exp (\imath \pi / 2)$ [53]. As a result, the transmitted field $\mathbf{E}_{\sigma}^{\mathrm{tr}}$, the intensity $S_{0}^{\mathrm{tr}}$, and the third Stokes parameters $S_{3}^{\text {tr }}$ are

$$
\begin{aligned}
& \mathbf{E}_{\sigma}^{\operatorname{tr}}(\rho)=E(\rho)\left[t_{p}(\rho) \hat{\boldsymbol{\rho}}+\sigma t_{s}(\rho) \hat{\boldsymbol{\varphi}}\right], \\
& S_{0}^{\operatorname{tr}}(\rho)=|E(\rho)|^{2}\left[\left|t_{p}(\rho)\right|^{2}+\left|t_{s}(\rho)\right|^{2}\right], \\
& S_{3}^{\operatorname{tr}}(\rho)=-2 \sigma|E(\rho)|^{2}\left|t_{p}(\rho) t_{s}(\rho)\right|,
\end{aligned}
$$

where $\rho=\sqrt{x_{2}^{2}+y_{2}^{2}}=f k_{\rho} / k$ and the angle of $30^{\circ}$ is given by the condition $\rho=0.5 f$ in $\mathrm{BFP}_{1}$. Equations (3) show that the transmitted beam at this angle is circularly polarized $S_{3}^{\mathrm{tr}} / S_{0}^{\mathrm{tr}} \approx-\sigma$ with the handedness being dependent on the spatial polarization distribution of the incident beam. This shows that Kelvin's chirality of the incident beam $K(\sigma)$ can perfectly couple to optical chirality $C$ of the transmitted beam in a simple cylindrically symmetric scenario.

\section{EXPERIMENT}

Figure 2 illustrates our experimental setup [54]. We transmit a Gaussian beam (wavelength $\lambda=532 \mathrm{~nm}$, linewidth $\Delta \lambda_{\text {FWHM }} \approx 4 \mathrm{~nm}$ ) through a linear polarizer (LP) and a qplate of charge $1 / 2[55,56]$. Depending on the relative angle between the LP and the q-plate, this results in a radially, azimuthally, or one of two spirally polarized doughnut-shaped beams $\mathrm{SP}_{1}(\sigma=+1)$ and $\mathrm{SP}_{2}(\sigma=-1)$. For comparison we also performed the measurements for linear $x$ - and $y$-polarized input beams. The investigated stratified medium on top of a glass substrate was fabricated by Iridian Spectral Technologies such that $t_{p} / t_{s} \approx \exp (l \pi / 2)$ at the angle of incidence of $30^{\circ}$ for the wavelength of $532 \mathrm{~nm}$. The numerical apertures (NA) of the two MOs were chosen to be larger than 0.5 to cover this angle. Additionally, we repeated the measurements on a bare glass substrate with refractive index of $n=1.52$ and thickness of $170 \mu \mathrm{m}$. Since the coherence length of our laser is only $\lambda^{2} /(n \Delta \lambda) \approx 47 \mu \mathrm{m}$, such a measurement provides reference data for the case where the sample does not introduce a phase shift between $p$ and $s$ polarizations [53]. We use two liquid crystal variable retarders and an LP to project the transmitted beam onto an arbitrary polarization state. Finally, we image the polarization resolved intensity distribution in the $\mathrm{BFP}_{2}$ onto a CMOS camera for full spatial Stokes vector reconstruction. 


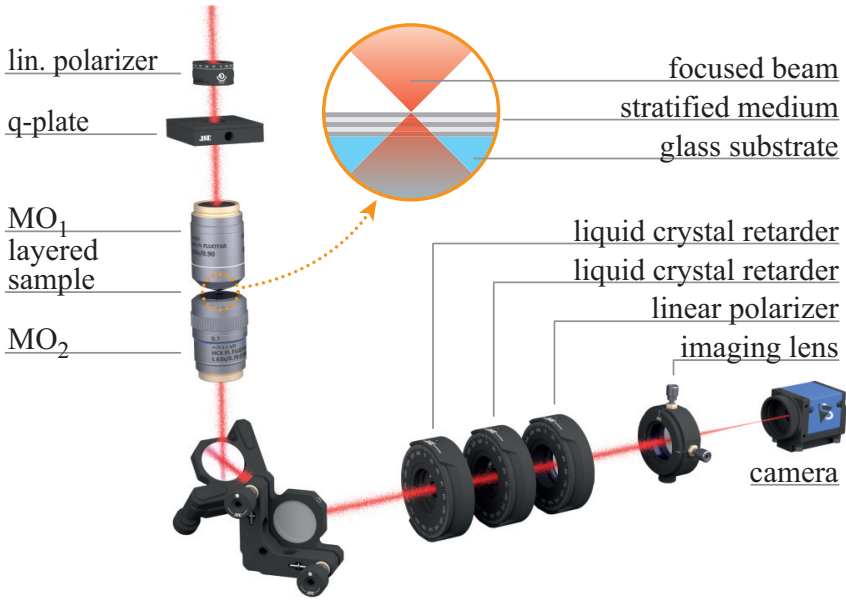

FIG. 2. Experimental setup. A linear polarizer and a q-plate of charge $1 / 2$ convert the incoming Gaussian beam into the desired mode. Two confocally aligned microscope objectives focus the incoming beam onto the sample and collimate it subsequently. Two liquid crystal variable retarders, a linear polarizer and a lens are used to perform a polarization resolved imaging of the back focal plane of the second microscope onto a camera.

First, Fig. 3(I) shows the measured input intensity distributions $S_{0}^{\text {in }}=\left[I_{\mathrm{RCP}}^{\text {in }}+I_{\mathrm{LCP}}^{\text {in }}\right]$, where $I_{\mathrm{RCP}}^{\text {in }}$ and $I_{\mathrm{LCP}}^{\text {in }}$ are the measured input intensities of the RCP and LCP polarizations. The red arrows depict the corresponding polarization patterns. Second, in Figs. 3(II) and 3(III) we plot the intensity distribution $S_{0}^{\mathrm{tr}}$ and the normalized third Stokes parameters $S_{3}^{\mathrm{tr}}=$ $\left[I_{\mathrm{RCP}}^{\mathrm{tr}}-I_{\mathrm{LCP}}^{\mathrm{tr}}\right] / \max \left(S_{0}^{\mathrm{tr}}\right)$ of the light transmitted through the stratified sample. The insets show the theoretical predictions. As expected, the radial and azimuthal beams do not generate CPL, while both spiral beams $\mathrm{SP}_{1}$ and $\mathrm{SP}_{2}$ strongly couple to CPL with the sign of the transmitted $S_{3}^{\text {tr }}$ depending on the spiral orientation of the incoming beam or, equivalently, on the sign of Kelvin's chriality $K(\sigma)$ [see Figs. 3(III)(a)-3(III)(d)]. In fact, for the angle of $30^{\circ}(\mathrm{NA}=0.5)$ we achieve almost perfect coupling to CPL with values of $S_{3}^{\text {tr }}$ approaching $\mp 1=$ $-\sigma$, as expected from Eqs. (3). For linear input polarizations, the cylindrical symmetry of the beam is broken, resulting in a fourfold pattern of CPL in Figs. 3(III)(e) and 3(III)(f). Nevertheless, since linearly polarized beams do not possess any Kelvin's chirality, their average generated $S_{3}^{\text {tr }}$ is zero. Finally, in Figs. 3(IV) and 3(V) we present the transmission measurements through the glass sample. Here in Figs. 3(V)(a)-3(V)(d) we observe only a residual pattern of $S_{3}^{\text {tr }}$ with zero average, resembling the measurements for the radial and azimuthal polarization through the stratified medium [Fig. 3(III)(a) and 3(III)(c)] or the glass sample [Figs. 3(V)(a) and 3(V)(c)]. This resemblance indicates that the residual $S_{3}^{\text {tr }}$ originates from the imperfections of the q-plate, as confirmed by the measurements of linear polarizations transmitted through the glass substrate in Figs. 3(V)(e) and 3(V)(f), which we performed without the q-plate.

\section{DISCUSSION AND CONCLUSION}

To comprehend the role of Kelvin's chirality $K$ it is worth discussing our result from the point of view of geometry, material composition, and conservation laws.
First, in chiral light-matter interactions it is not exclusively the chirality of matter that couples to optical chirality $C$, but essentially the geometrical chirality of the whole experiment. For instance, structurally achiral planar metamolecules show chiroptical response at normal incidence, if the mirror symmetry is broken by the heterogeneous material composition of their constituents [57,58]. Additionally, chiroptical effects also appear if a planar structure and the $k$ vector of an obliquely incident CPL form a geometrically chiral arrangement [59]. Similarly, linear dipoles can emit chiral light, when appropriately positioned close to an optical waveguide $[60,61]$. In this regard, $K$ ensures $\hat{\mathrm{P}}$-symmetry breaking at the level of beam geometry [31].

Second, we may ask which physical conservation laws permit generation of CPL in a cylindrically symmetric system? Helicity - the projection of spin onto the propagation direction-characterizes the handedness of a beam [17-21,42-49]. Helicity is only preserved in electromagnetically dual (impedance matched) conditions, equivalent to $t_{p}=t_{s}$ at all angles for planar systems $[42,45]$. Neither the stratified medium nor the glass substrate preserve helicity. Our focused beam acquires circular polarization upon transmission through the stratified medium for the same physical reason that obliquely incident plane-wave CPL at an air-glass interface acquires elliptical polarization in ellipsometry measurements, i.e., the difference of the two Fresnel coefficients.

Third, we construct the spiral beams with $K \neq 0$ by a superposition of a radially and an azimuthally polarized beam. Neither of these beams alone possesses Kelvin's chirality. However, the spatial polarization distributions of radial and azimuthal beams are $\hat{\mathrm{P}}$ even and $\hat{\mathrm{P}}$ odd, respectively, relative to a reflection plane that contains the $z$ axis, which breaks the $\hat{\mathrm{P}}$ symmetry of their superposition [31]. Surprisingly, a $\hat{\mathrm{P}}$-even spatial polarization distribution of the radial beam does not violate the parity odd transformation of $\mathbf{E}$ itself, which holds at each point of space such that $\hat{\mathrm{P}}\{\mathbf{E}(x, y, z)\}=-\mathbf{E}(-x,-y,-z)$ (see also Fig. 1(c) in [41]). Moreover, we consider the geometry of spiral beams along with their direction of propagation, which renders them asymmetric under time reversal $(\hat{T})$ and symmetric under combined $\hat{P} \hat{T}$ inversion. Previously, Barron defined these transformation properties as "false chirality" in molecular systems [2-4] and they are still a subject of active research [16]. At the same time, corkscrew wave fronts of linearly polarized Laguerre-Gaussian beams are asymmetric under $\hat{\mathrm{P}}$ and symmetric under $\hat{\mathrm{T}}$ reversal, respectively, rendering them "truly chiral" [31]. We envision that further classification of $K$ and transformation properties of structured beams may be necessary.

Fourth, parity asymmetric spatial distribution of an electric field can manifest itself in amplitude, phase, polarization, or a combination thereof. For instance, in our experiment $K$ is related exclusively to polarization. Our setup is insensitive to the phase distribution and any imprint of an inhomogeneous phase-including optical vortices-would not alter the distribution of $C$ in Fig. 3. Moreover, there is no general relation between $K$ and optical vortices or beams carrying orbital angular momentum (OAM) [32-39]. Similarly, there is no general relation between spin angular momentum (SAM) density and optical chirality $C[13,18,19,43,46,62]$. In the experiment, the incident spiral beams possess $K \neq 0$ and zero SAM, OAM and $C$, while the transmitted beams have nonzero 
(a) radial
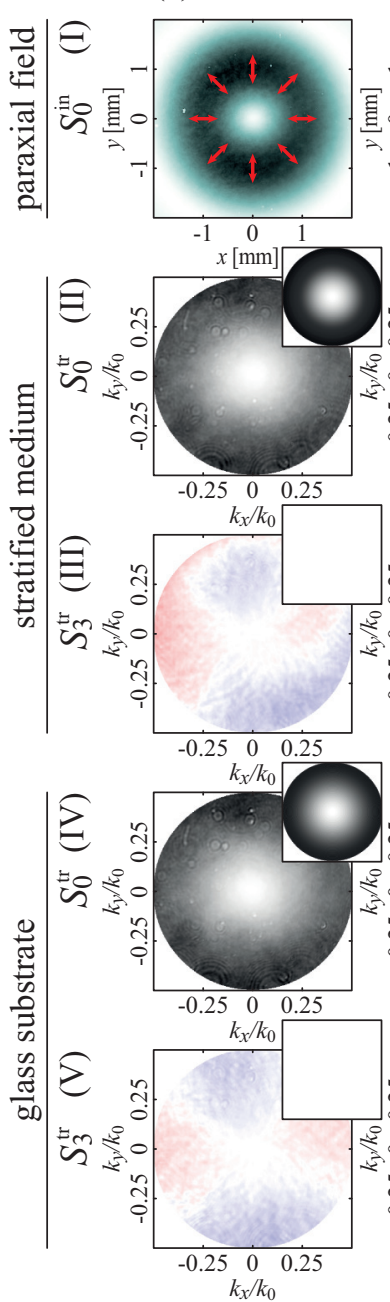

(b) $\mathrm{SP}_{1}$

(c) azimuthal

(d) $\mathrm{SP}_{2}$

(e) linear-x
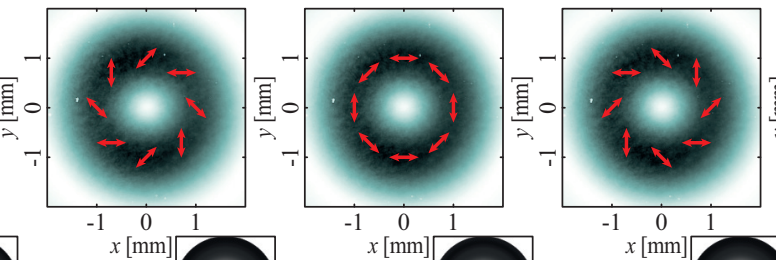

(f) linear-y
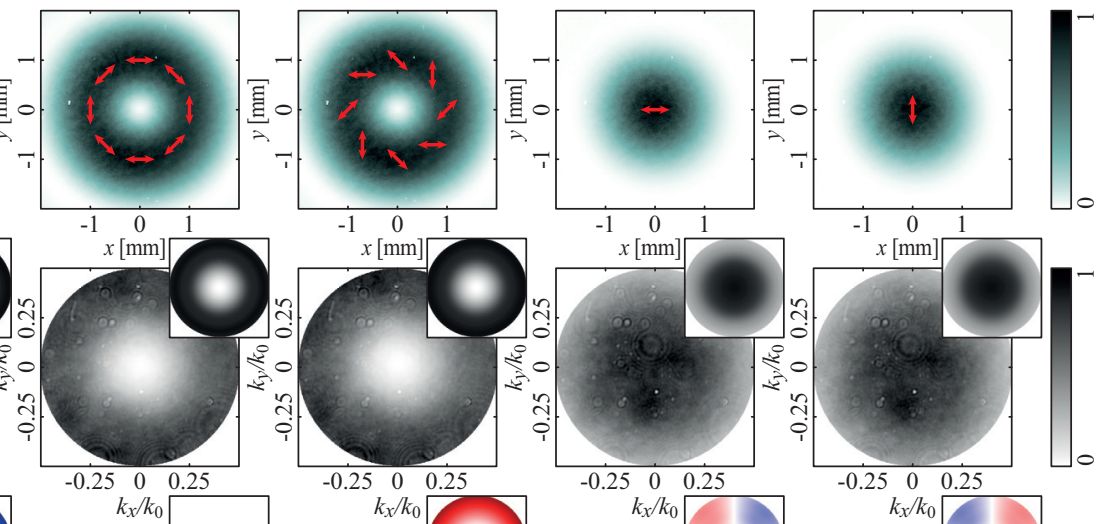
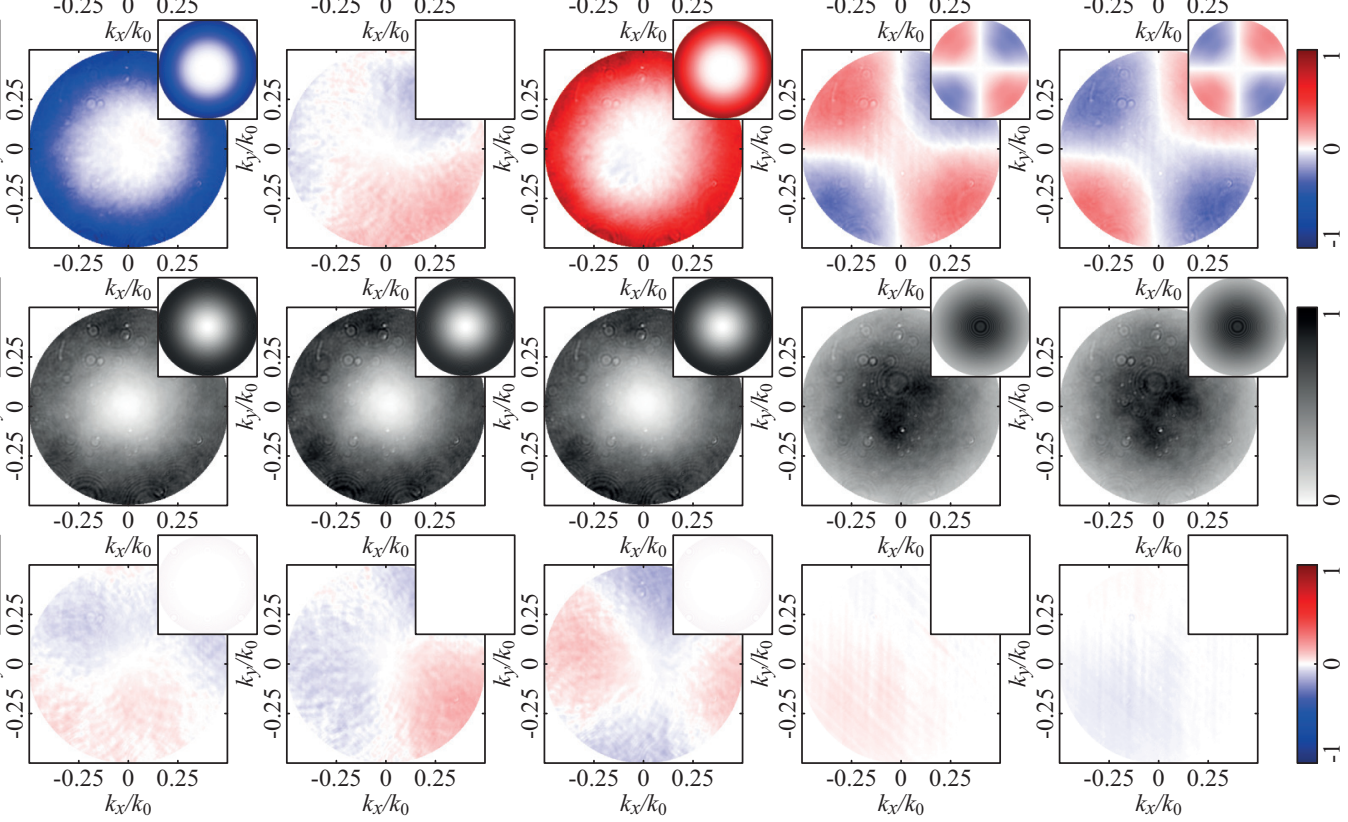

FIG. 3. Measurements for six input polarizations: (a) radial, (b) spiral $\mathrm{SP}_{1}$, (c) azimuthal, (d) spiral $\mathrm{SP}_{2}$, (e) linear $x$, and (f) linear $y$. (I) Intensity distributions before entering the first microscope objective. The local polarization state is indicated by red arrows. Stokes parameters $S_{0}^{\mathrm{tr}}(\mathrm{II})$ and $S_{3}^{\mathrm{tr}}(\mathrm{III})$, measured in transmission through the stratified medium. Theoretical counterparts are plotted as insets. $S_{0}^{\mathrm{tr}}(\mathrm{IV})$ and $S_{3}^{\mathrm{tr}}(\mathrm{V})$ correspond to transmission through a glass substrate.

SAM, OAM, $C$, and $K$ [36,40,41]. However, specific cases of SAM to OAM conversion [63,64] also serve as illustrations of coupling between $C$ and $K-$ a reverse phenomenon to the one we have considered. Additionally, some works focusing on OAM also illustrate interaction between $K$ and chirality of matter [32-39].

Finally, Kelvin's chirality of optical beams, similarly to chirality of matter, is a function of a geometrical shape, which is independent of its optical manifestations and does not rely on any measurable observable. For instance, an unfocused spiral beam transmitted through the stratified sample would not acquire optical chirality since $t_{p}=t_{s}$ at normal incidence. Quantification of $K$ is therefore as elusive a task as quantification of chirality of matter, for which many attempts have been made, but no universal measure has been established to date [5-11].

In conclusion, we presented an experiment where the geometrical chirality of an optical beam, termed here as Kelvin's chirality and manifested as parity asymmetric spatial polarization distribution, couples to optical chirality upon transmission of a focused beam through a planar medium in a cylindrically symmetric scenario. We elucidated the underlying mechanism of chiral light-matter interaction by symmetry, material composition, helicity conservation laws, and a simple analytical model. Our results emphasize that spatially inhomogeneous phase and polarization profiles of structured light beams constitute an important degree of freedom in chiral light-matter interactions beyond optical chirality.

\section{ACKNOWLEDGMENTS}

R.A. acknowledges the support of the Alexander von Humboldt Foundation through the Feodor Lynen Return Fellowship. R.A. and R.W.B. acknowledge support through the Natural Sciences and Engineering Research Council of Canada, the Canada Research Chairs program, and the Canada First Research Excellence Fund. R.W.B. acknowledges support under US Office of Naval Research MURI award N00014-20-1-2558.

S.N. and J.S.E. contributed equally to this work. 
[1] W. T. Kelvin, The Molecular Tactics of a Crystal (Oxford Clarendon, Oxford, 1894).

[2] L. D. Barron, Chem. Phys. Lett. 123, 423 (1986).

[3] L. D. Barron, Chem. Soc. Rev. 15, 189 (1986).

[4] L. D. Barron, Chirality 24, 879 (2012).

[5] A. B. Buda and K. Mislow, J. Am. Chem. Soc. 114, 6006 (1992).

[6] A. B. Buda, T. A. der Heyde, and K. Mislow, Angew. Chem. Int. Ed. Engl. 31, 989 (1992).

[7] A. Rassat and P. W. Fowler, Chem. Eur. J. 10, 6575 (2004).

[8] P. W. Fowler, Symmetry Culture Sci. 16, 321 (2005).

[9] E. Efrati and W. T. M. Irvine, Phys. Rev. X 4, 011003 (2014).

[10] I. Fernandez-Corbaton, M. Fruhnert, and C. Rockstuhl, Phys. Rev. X 6, 031013 (2016).

[11] P. Gutsche, X. Garcia-Santiago, P. I. Schneider, K. M. McPeak, M. Nieto-Vesperinas, and S. Burger, Symmetry 12, 158 (2020).

[12] Y. Tang and A. E. Cohen, Phys. Rev. Lett. 104, 163901 (2010).

[13] K. Y. Bliokh and F. Nori, Phys. Rev. A 83, 021803(R) (2011).

[14] M. M. Coles and D. L. Andrews, Phys. Rev. A 85, 063810 (2012).

[15] D. L. Andrews and M. M. Coles, Opt. Lett. 37, 3009 (2012).

[16] K. Y. Bliokh, Y. S. Kivshar, and F. Nori, Phys. Rev. Lett. 113, 033601 (2014).

[17] M. Nieto-Vesperinas, Philos. Trans. R. Soc. A 375, 20160314 (2017).

[18] R. P. Cameron, J. Götte, S. M. Barnett, and A. M. Yao, Philos. Trans. R. Soc. A 375, 20150433 (2017).

[19] F. Crimin, N. Mackinnon, J. Götte, and S. Barnett, Appl. Sci. 9, 828 (2019).

[20] L. V. Poulikakos, J. A. Dionne, and A. Garca-Etxarri, Symmetry 11 (2019).

[21] N. Mackinnon, J. Opt. 21, 125402 (2019).

[22] V. K. Valev, J. J. Baumberg, C. Sibilia, and T. Verbiest, Adv. Mater. 25, 2517 (2013).

[23] D. S. Bradshaw, J. M. Leeder, M. M. Coles, and D. L. Andrews, Chem. Phys. Lett. 626, 106 (2015).

[24] P. Lodahl, S. Mahmoodian, S. Stobbe, A. Rauschenbeutel, P. Schneeweiss, J. Volz, H. Pichler, and P. Zoller, Nature (London) 541, 473 (2017).

[25] R. W. Woody, in Biochemical Spectroscopy, Methods in Enzymology Vol. 246 (Academic, New York, 1995), pp. 34-71.

[26] L. D. Barron, Molecular Light Scattering and Optical Activity, 2nd ed. (Cambridge University Press, Cambridge, 2004).

[27] M. V. Berry and M. R. Dennis, Proc. R. Soc. London Ser. A 457, 2251 (2001).

[28] J. Leach, M. R. Dennis, J. Courtial, and M. J. Padgett, Nature (London) 432, 165 (2004).

[29] H. Larocque, D. Sugic, D. Mortimer, A. J. Taylor, R. Fickler, R. W. Boyd, M. R. Dennis, and E. Karimi, Nat. Phys. 14, 1079 (2018).

[30] T. Bauer, P. Banzer, E. Karimi, S. Orlov, A. Rubano, L. Marrucci, E. Santamato, R. W. Boyd, and G. Leuchs, Science 347, 964 (2015).

[31] Here parity inversion relates to a mirror reflection of the spatial structure of a beam relative to a plane that contains the propagation direction. This should not be confused with the fact that electric field is a polar vector that locally obeys $\hat{\mathrm{P}}\{\mathbf{E}(x, y, z)\}=$ $-\mathbf{E}(-x,-y,-z)$.

[32] K. Toyoda, K. Miyamoto, N. Aoki, R. Morita, and T. Omatsu, Nano Lett. 12, 3645 (2012).

[33] G. K. L. Wong, M. S. Kang, H. W. Lee, F. Biancalana, C. Conti, T. Weiss, and P. S. J. Russell, Science 337, 446 (2012).

[34] Y. Gorodetski, A. Drezet, C. Genet, and T. W. Ebbesen, Phys. Rev. Lett. 110, 203906 (2013).

[35] K. A. Forbes and D. L. Andrews, Opt. Lett. 43, 435 (2018).

[36] S. Nechayev, J. S. Eismann, G. Leuchs, and P. Banzer, Phys. Rev. B 99, 075155 (2019).

[37] P. Woźniak, I. D. Leon, K. Höflich, G. Leuchs, and P. Banzer, Optica 6, 961 (2019).

[38] K. A. Forbes and D. L. Andrews, Phys. Rev. A 99, 023837 (2019).

[39] K. A. Forbes and D. L. Andrews, Phys. Rev. Res. 1, 033080 (2019).

[40] J. S. Eismann, M. Neugebauer, and P. Banzer, Optica 5, 954 (2018).

[41] S. Nechayev and P. Banzer, Phys. Rev. B 99, 241101(R) (2019).

[42] I. Fernandez-Corbaton, X. Zambrana-Puyalto, and G. MolinaTerriza, Phys. Rev. A 86, 042103 (2012).

[43] R. P. Cameron, S. M. Barnett, and A. M. Yao, New J. Phys. 14, 053050 (2012).

[44] S. M. Barnett, R. P. Cameron, and A. M. Yao, Phys. Rev. A 86, 013845 (2012).

[45] I. Fernandez-Corbaton, X. Zambrana-Puyalto, N. Tischler, X. Vidal, M. L. Juan, and G. Molina-Terriza, Phys. Rev. Lett. 111, 060401 (2013).

[46] K. Y. Bliokh, A. Y. Bekshaev, and F. Nori, New J. Phys. 15, 033026 (2013).

[47] M. Nieto-Vesperinas, Phys. Rev. A 92, 023813 (2015).

[48] M. Nieto-Vesperinas, J. Opt. 19, 065402 (2017).

[49] I. Fernandez-Corbaton and C. Rockstuhl, Phys. Rev. A 95, 053829 (2017).

[50] L. Novotny and B. Hecht, Principles of Nano-Optics (Cambridge University Press, Cambridge, 2012).

[51] Q. Zhan and J. R. Leger, Opt. Express 10, 324 (2002).

[52] S. Nechayev, J. S. Eismann, M. Neugebauer, and P. Banzer, ACS Photonics 7, 581 (2020).

[53] P. Yeh, A. Yariv, and C.-S. Hong, J. Opt. Soc. Am. 67, 423 (1977).

[54] P. Banzer, U. Peschel, S. Quabis, and G. Leuchs, Opt. Express 18, 10905 (2010).

[55] L. Marrucci, C. Manzo, and D. Paparo, Phys. Rev. Lett. 96, 163905 (2006).

[56] H. Larocque, J. Gagnon-Bischoff, F. Bouchard, R. Fickler, J. Upham, R. W. Boyd, and E. Karimi, J. Opt. 18, 124002 (2016).

[57] P. Banzer, P. Woniak, U. Mick, I. De Leon, and R. W. Boyd, Nat. Commun. 7, 1 (2016).

[58] S. Nechayev, R. Barczyk, U. Mick, and P. Banzer, ACS Photonics 6, 1876 (2019).

[59] C. W. Bunn, Chemical Crystallography (Oxford University Press, Oxford, 1945).

[60] M. Joos, C. Ding, V. Loo, G. Blanquer, E. Giacobino, A. Bramati, V. Krachmalnicoff, and Q. Glorieux, Phys. Rev. Appl. 9, 064035 (2018). 
[61] M. Neugebauer, P. Banzer, and S. Nechayev, Sci. Adv. 5, aav7588 (2019).

[62] H. Li, V. Rodriguez-Fajardo, P. Chen, and A. Forbes, Phys. Rev. A 102, 063533 (2020).
[63] Y. Zhao, J. S. Edgar, G. D. M. Jeffries, D. McGloin, and D. T. Chiu, Phys. Rev. Lett. 99, 073901 (2007).

[64] K. Y. Bliokh, F. J. Rodríguez-Fortuño, F. Nori, and A. V. Zayats, Nat. Photonics 9, 796 (2015). 